\title{
The Emergency Strategic Clinical Network (ESCN) quality improvement and innovation forum
}

\section{Patrick McLane, Eddy Lang}

Evidence-based research and quality improvement work are pivotal to health systems meeting their goals. Translating findings and disseminating innovative practices to new settings occurs in part through knowledge translation events, such as conferences and workshops.

The Emergency Strategic Clinical Network ${ }^{\mathrm{TM}}$ (ESCN) Quality Improvement and Innovation forum fills a gap between local and national events. It is devoted to sharing methods and results of emergency department projects in Alberta among those working in emergency care. 2020 was the second consecutive year the ESCN has held this one day event.

The event provides an opportunity for those working on quality improvement in emergency medicine to network with one another, share innovative projects, share know how and translate promising works to new settings. In addition, the event provides an opportunity to identify projects for potential development through local, provincial, or even national opportunities.

Each year, over 30 teams have presented their projects through a mix of plenary oral and lightning oral formats. All abstracts submitted to the event are accepted, with abstract rankings determining which projects are selected for plenary oral presentations. Among the many topics presented, mental health and addictions, Choosing Wisely ${ }^{\mathrm{TM}}$ initiatives, pediatric pain control, clinical skill improvement projects, Indigenous health, staffing, and nurse initiated protocols have featured prominently. Methods have included formal quality improvement projects, systematic reviews, qualitative research, observational studies and health records reviews.

Strong participation shows the value practitioners see in the forum. In 2019, the forum was attended by approximately 90 educators, managers, nurses, physicians and researchers from across Alberta. In 2020, 82 delegates attended in person, while 33 attended virtually. Travel for the 2020 forum was generously supported by the College of Physicians and Surgeons of Alberta.

We are pleased to partner with the Canadian Journal of Emergency Nursing to make abstracts from the event widely available. Individual presenters have had the option of submitting their abstracts for publication in CJEN. In some instances, abstracts have already been published through other conferences and so could not be submitted to CJEN.

The findings presented in the abstracts are solely the work of the submitting authors. The ESCN does not guarantee the accuracy of any reported information. The views expressed in the abstracts are solely the views of the authors and do not represent the ESCN or Alberta Health Services.

Correspondence to: emergencyscn@ahs.ca 